\title{
Breast cancer trend in Taiwan
}

\section{Mini review}

Breast cancer is the most common cancer among women, according to the cancer registration report of the Ministry of Health and Welfare (MOHW), in 2014, ${ }^{1}$ up to 11,976 women suffered from breast cancer, which meant 33 women suffered from breast cancer daily. The latest statistical data revealed that, in Taiwan, the ranking of breast cancer and cervical cancer incidence rate in 2014 were the top (70.74 per 100,000 population) and the eighth ( 8.5 per 100,000 population), respectively; while compared with the incident numbers in $2013,{ }^{2}$ they were 695 cases in addition and 127 cases in decline, respectively; when it comes to incidence rate, it was $2 \%$ increased and $10 \%$ declined, respectively. The number of breast cancer increased more significantly. In 2014, the mortality rate of breast cancer ranked the fourth, the death toll was 2,071, which means around 5.67 women died from breast cancer each day. Due to the westernized lifestyle and dietary habits, the age of breast cancer onset in Taiwan gets younger (Figure 1), and is about 10 years younger than that in the western countries. According to the statistics (Figure 2), the rate of early stage (stages $0-2$ ) breast cancer cases diagnosed by screening was $86 \%$, whereas the rate of those not diagnosed by screening was $56 \%$. Regular screening assists in recognizing early stage of breast cancer, and early treatment increases the remission rate, therefore, the five-year survival rate of screened patients reached higher than $90 \%$, whereas the five-year survival rate of unscreened patients suffered from breast cancer diagnosed at late stage (stage 4) was only $27.8 \%$. Mammography is the process of using low-energy X-rays (about $0.7 \mathrm{mSv}$ ) to examine human breast for screening calcification and stage 0 (carcinoma in situ) of breast cancer. ${ }^{3}$ A randomized trial with large scale mammography screening of breast cancer in 1977 has adopted the spilt screen design with inviting one group for periodical mammography while the other group was not invited. When the trial was ended in 1985, the controlled group was provided with one screening to balance the prevalence screening cases of screening group and lessen bias. After following up for several years, it showed a significant difference in mortality rate. The mortality rate of breast cancer in women with periodical screening declines up to 30 percent. ${ }^{4}$ Nowadays, according to the medical evidence, the western countries promote large scale screening services for women as well as subsidies of breast x-ray once every two years for patients aged 45-69 or patients aged 40-44 with family history of breast cancer (which means anyone of the patient's grandmothers, mother, daughter(s) or sister(s) has ever suffered from breast cancer) to protect the health of women's breasts.

In consideration of the influences of cancer on medical expenses and national productivity, Taiwan has promoted the national cancer prevention projects since 2004, and now it comes to the third period. The targets of the project are early stage cancers with high remission rate such as breast cancer, cervical cancer, oral cancer and colon cancer. Four strategies were introduced such as improving people's ability of cancer prevention, expanding and carrying out cancer screening as well as enhancing screening rate and quality, improving appropriate care as well as constructing a database of cancer screening, incidence and monitoring. By decreasing the rate of chewing betel nuts, increasing the screening rate of all kinds of cancer, increasing tracking
Volume 6 Issue 2 - 2017

\author{
Ying Ping Chen, Yin-Wen Lu, Chih-Ching Yang \\ Department of Planning, Ministry of Health and Welfare, Taiwan
}

Correspondence: Chih-Ching Yang, Ministry of Health and Welfare, No.488, Sec. 6, Zhongxiao E. Rd., Nangang Dist., Tai-pei City II5, Taiwan, Email mdyangcc@mohw.gov.tw

Received: November 01, 2017 | Published: November 13, 2017

rate of positive screening cases, increasing medical treatment rate for newly diagnosed cancer patients within 3-month, and increasing the quality of cancer treatment by comparing our quality index with international ones in the relevant database, it is expected that in 20092025 , our domestic cancer mortality rate could decline from 132.5 per 100,000 population to 119 per 100,000 population. Figure 3 shows that, since 2004, after 10-year effort, the breast cancer incidence rate still increased slightly. It may be influenced by the risk factors includes age, early menarche, late menopause, family history and unhealthy lifestyle, ${ }^{5}$ besides, the gradually increased screening rate of mammography also assists in early diagnosis and treatment of breast cancer. Therefore, it showed slightly change in breast cancer mortality rate in recent 10 years. Furthermore, the follow-up completion rate of breast cancer screening for positive cases has also been increased to $89.5 \%$.

When studying the breast cancer in detail with 5-year incidence rate and mortality rate of the 6 municipalities (Figures 4) (Figure 5), it showed that after 2010, the incidence rate of the 6 municipalities kept increasing and the incidence rates of breast cancer in the 5 municipalities were higher than the average domestic level besides Tainan City, whereas that of Taichung City ranked 5 out of 6 municipalities. When it comes to breast cancer mortality rate, both domestic data and that of the 6 municipalities tended to decline, especially in 2013-2014, but it showed the least degree of declining in Taichung City. In 2014, the breast cancer mortality rate of Taichung City was not only higher than the domestic average but stood in the first place of the 6 municipalities. Figure 6 shows that in 2012-2014, the mammography screening rate of mid-Taiwan medical facilities was lower than other regions, which might be the reason that the breast cancer incidence rate of Taichung City was lower than that of the other 5 municipalities, by the way, the reason of higher mortality rate might come from low breast cancer mammography screening rate, late diagnosis of breast cancer or the quality of medical care needs to be enhanced, while the exact reason was not yet understood without further data collection. This example showed that data analysis assists in policy making and management and furthermore, we could improve our plan about cancer prevention according to the different trends of the nations, the cities and counties such as promoting health literacy of cancer prevention, taking care of female citizens and encouraging women with screening to increase the screening rate in order to gain the best prevention effect. 


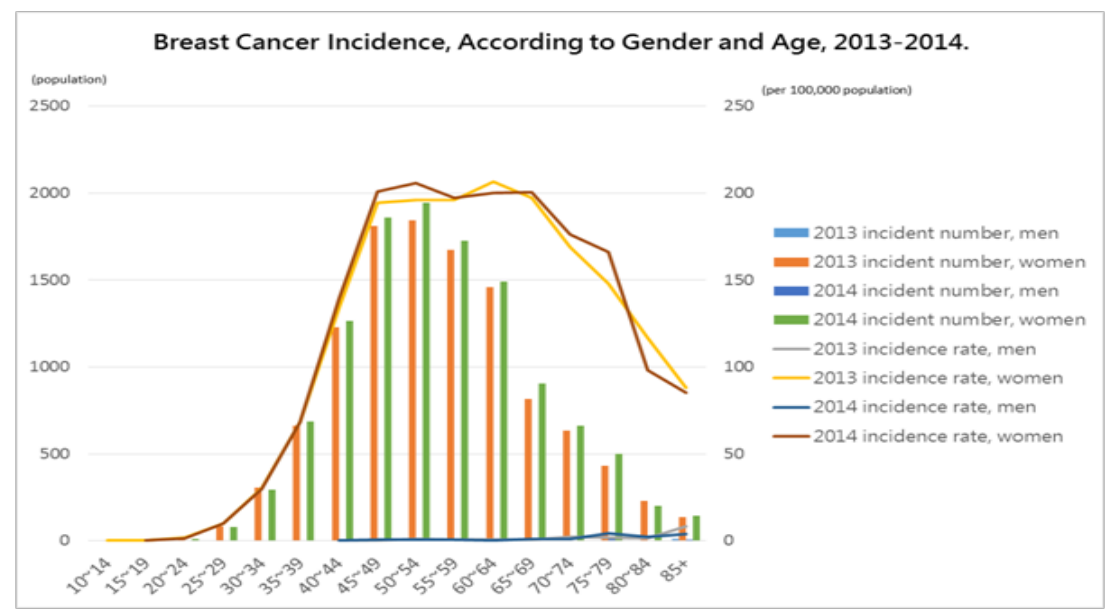

Figure I Breast Cancer Incidence,According to Gender and Age, 20I3-20I4.

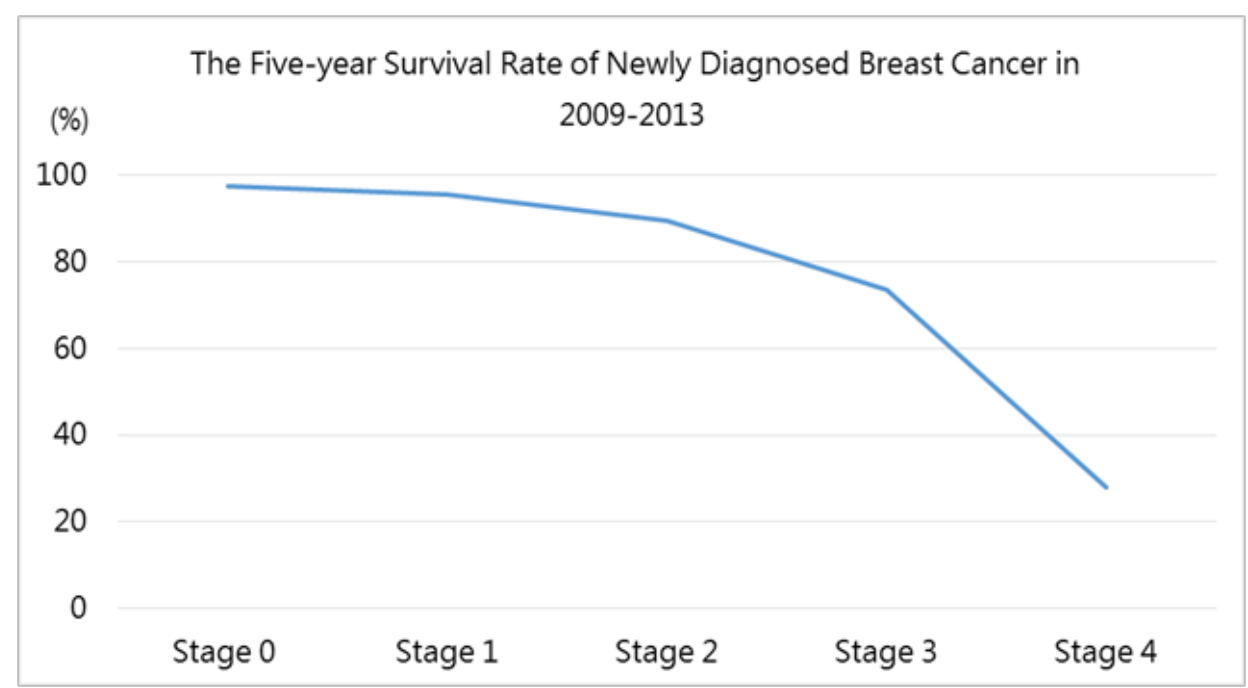

I. Source:Taiwan nationwide cancer registration system (including carcinoma in situ)

II. Analyzing the five-year survival rate data from 2009-20I3 NHI claims (tracking to 2013)

Figure 2 The five-year Survival Rate of Newly Diagnosed Breast Cancer in 2009-20I3.

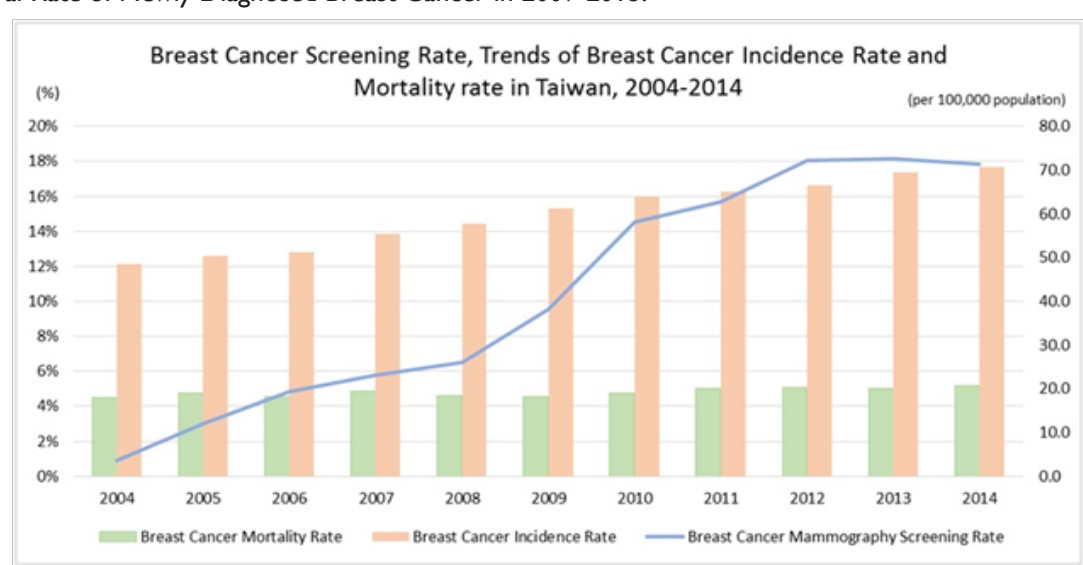

Figure 3 Breast cancer screening rate, trends of breast cancer incidence rate and mortality rate in Taiwan, 2004-20I4 


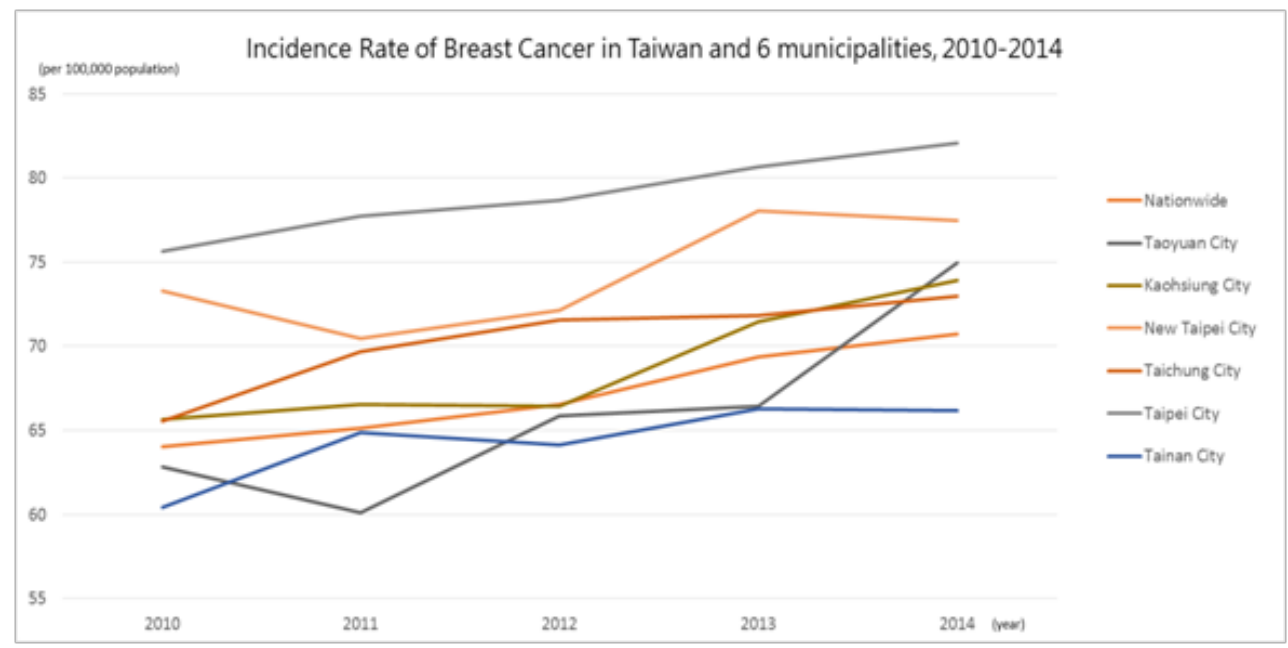

Figure 4 Incidence rate of breast cancer in Taiwan and 6 municipalities, 2010-20I4.

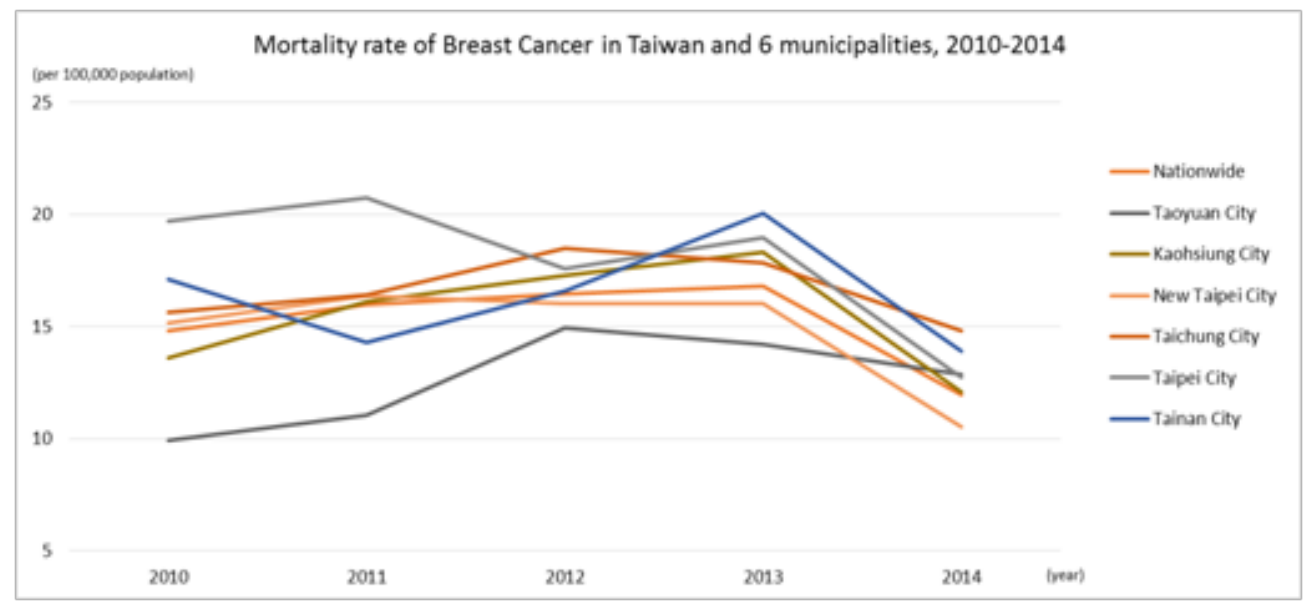

Figure 5 Mortality rate of Breast Cancer in Taiwan and 6 municipalities, 20I0-20I4.

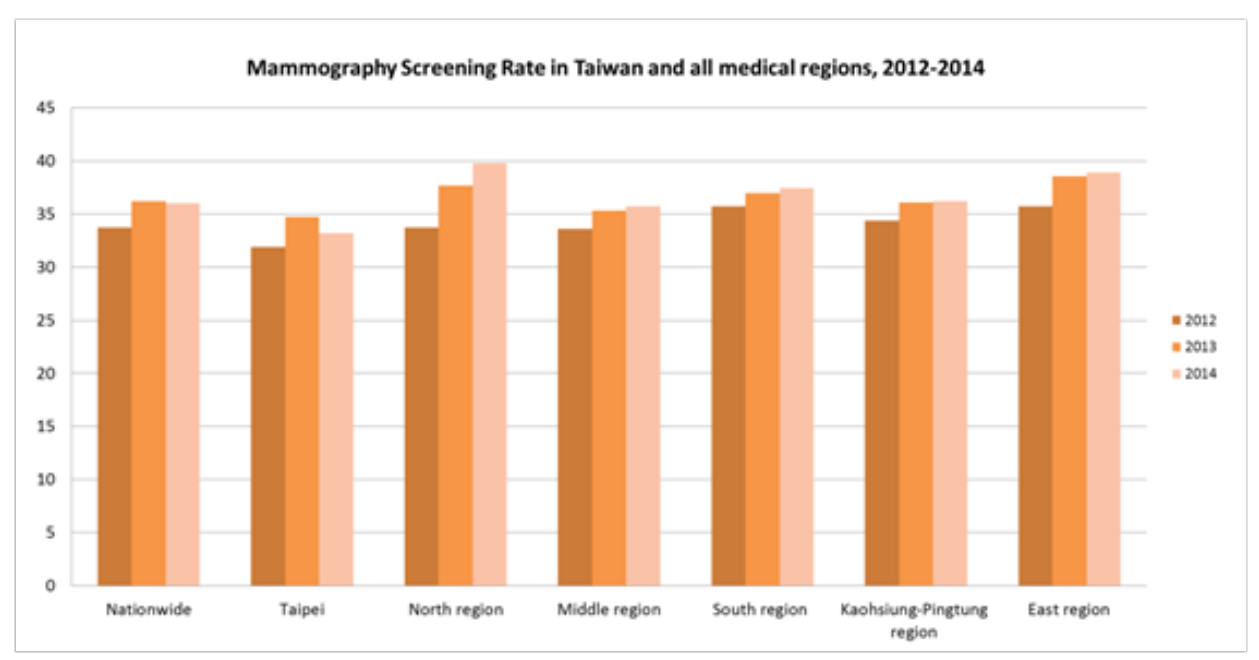

Figure 6 Mammography Screening Rate in Taiwan and all medical regions, 2012-2014. 


\section{Acknowledgements}

None.

\section{Conflict of interest}

The author declares no conflict of interest

\section{References}

1. Cancer Registration Report 2014, HPA, the MOHW.
2. Cancer Registration Report 2013, HPA, the MOHW.

3. Blausen gallery. Wikiversity Journal of Medicine; 2014.

4. Tabár L, Vitak B, Chen TH, et al. Swedish Two-County Trial: Impact of Mammographic screening on breast cancer mortality during 3 decades. Radiology. 2011;260(3):658-663.

5. How preventable is breast cancer? American Institute for Cancer Research (AICR), USA. 